\title{
DERMATOSE BOLHOSA IGA LINEAR EM DOENTE COM COLITE ULCEROSA - UMA ASSOCIAÇÃO RARA MAS ESTABELECIDA
}

\author{
António Fernandes Massa', Catarina Moreira ${ }^{2 *}$, Cármen Lisboa ${ }^{3, *}$, Paulo Santos ${ }^{3}$, Joana Pardal ${ }^{4}$, Filomena Azevedo 5 \\ IInterno do Internato de Dermatovenereologia/Resident, Dermatology and Venereology, Serviço de Dermatologia, \\ Centro Hospitalar V. N. Gaia/Espinho, EPE, Vila Nova de Gaia, Portugal \\ 2Interna do Internato Complementar de Dermatovenerelogia/Resident, Dermatology and Venereology, Serviço de \\ Dermatologia e Venereologia, C.H. de S. João EPE, Porto, Portugal \\ ${ }^{3}$ Assistente Hospitalar Graduado/Graduated Consultant, Serviço de Dermatologia e Venereologia, C.H. de S. João \\ EPE, Porto, Portugal \\ ${ }^{4}$ Interna do Internato Complementar de Anatomia Patológica/Resident, Pathology, Serviço de Anatomia Patológica, C.H. \\ de S. João EPE Porto, Portugal \\ ${ }^{5}$ Chefe de Serviço e Directora de Serviço/Consultant Chief and Head, Serviço de Dermatologia e Venereologia, C.H. \\ de S. João EPE, Porto, Portugal \\ *Faculdade de Medicina, Universidade do Porto/Medical School, O'Porto University, Portugal
}

\begin{abstract}
RESUMO - A associação da dermatose bolhosa IgA linear (DBAL) com colite ulcerosa (CU) está descrita, embora a sua origem desta associação não esteja bem definida. Descreve-se o caso de uma doente de 41 anos, com CU diagnosticada há 6 anos e agravamento há 2 meses, referenciada devido a bolhas tensas, pruriginosas, dispersas no tegumento, com 1 mês de evolução. Referidos antecedentes de diabetes gestacional, depressão reactiva e hipertensão arterial. Medicada com mesalazina desde há 1,5 anos, bisoprolol, mexazolam e drospirenona/etinilestradiol. A biopsia cutânea mostrou bolha na junção dermoepidérmica, com infiltrado inflamatório polimórfico dérmico. A imunorreactividade linear de IgA confirmou o diagnóstico de DBAL. Foi iniciada prednisolona e dapsona com boa resposta clínica. A etiologia desta associação poderá dever-se à reactividade cruzada do idiotipo IgA presente na CU contra os antigénios dermoepidérmicos. Não se pode excluir totalmente a imputabilidade da mesalazina na indução de uma forma de DBAL associada a fármacos.
\end{abstract}

PALAVRAS-CHAVE - Dermatose bolhosa IgA linear; Vesicobolhosa, Doenças da pele; Colite ulcerosa; Doença inflamatória intestinal.

\section{LINEAR IMMUNOGLOBULIN A BULLOUS DERMATOSIS IN A PATIENT WITH ULCERATIVE COLITIS - A RARE BUT ESTABLISHED ASSOCIATION}

ABSTRACT - The association of linear IgA bullous dermatosis (LABD) with ulcerative colitis (UC) is defined, although the etiology of this association is still unclear. We report the case of a 41 year-old female patient with UC diagnosed 6 years ago with a crisis 2 months ago, referred for pruriginous and tense bullae with 1 month of evolution. Her past medical history was relevant for gestational diabetes, reactive depression and hypertension. She was medicated with mesalazine for 1,5 years, bisoprolol, mexazolam and drospirenone/ethinyl estradiol. Cutaneous biopsy showed a bulla on the dermoepidermal junction (DEJ) and a polymorphous inflammatory dermal infiltrate. Linear immunoreactivity for IgA on the DEJ confirmed the diagnosis of LABD. Prednisolone and dapsone were initiated with good clinical response, maintained after six months of follow-up. The aetiology of this association remains unknown, being hypothesised cross-reactivity of IgA idiotype from UC with the dermoepidermal antigens. Mesalazine imputability cannot be surely ruled 


\title{
Caso Clínico
}

out as the cause of LABD in this patient.

KEY-WORDS - Linear IgA bullous dermatosis; Skin diseases, Vesiculobullous; Ulcerative colitis; Inflammatory bowel disease.

\begin{abstract}
Conflitos de interesse: Os autores declaram não possuir conflitos de interesse.
No conflicts of interest.

Suporte financeiro: O presente trabalho não foi suportado por nenhum subsídio ou bolsa.

No sponsorship or scholarship granted.

Direito à privacidade e consentimento escrito / Privacy policy and informed consent: Os autores declaram que pediram consentimento ao doente para usar as imagens no artigo. The authors declare that the patient gave written informed consent for the use of its photos in this article.
\end{abstract}

Recebido/Received - Agosto/August 2014; Aceite/Accepted - Setembro/September 2014

Por decisão dos autores, este artigo não foi redigido de acordo com os termos do novo Acordo Ortográfico.

\section{Correspondêneico:}

Dr. António F. Massa

Serviço de Dermatologia

Centro Hospitalar V.N.Gaia/Espinho, EPE

R. Conceição Fernandes

4430 Vila Nova de Gaia, Portugal

E-mail: antoniofmassa@gmail.com

\section{INTRODUÇÃO}

O atingimento multiorgânico das doenças autoimunes é frequente, assim como a associação de diferentes doenças autoimunes num mesmo indivíduo.

A associação entre doenças bolhosas autoimunes subepidérmicas e outras doenças é rara, estando estabelecida a associação do penfigóide bolhoso (PB) com doença neurológica'.

Estão, ainda, descritos casos de associação de doenças bolhosas subepidérmicas autoimunes a doença inflamatória intestinal (DII) nomeadamente com a dermatose bolhosa lgA linear (DBAL), o PB, o penfigóide das membranas mucosas e o penfigóide gestacional. Shipman $A R$ e col. ${ }^{2}$ concluíram que a associação mais frequente foi a de DBAL com $\mathrm{CU}$, mesmo sendo a DBAL a mais rara das doenças bolhosas.

\section{CASO CLÍNICO}

Descrevemos o caso de uma doente do sexo feminino, de 41 anos, com CU diagnosticada há 6 anos. Foi referenciada à consulta de Dermatologia por bolhas pruriginosas na face interna das coxas, com 1 mês de evolução. Referia agravamento da CU cerca de 1 mês antes do início da dermatose. Ao exame objectivo, observavam-se bolhas tensas na sua maioria, de conteúdo cítrico, em placas de base eritematosa, com dimensão compreendida entre $0,5 \mathrm{~cm}$ e $3 \mathrm{~cm}$, dispersas no abdómen, púbis, área supraescapular direita, face interna das coxas, região maleolar e calcâneos. Sem sinal de Nikolsky. Não havia atingimento da mucosa oral ou da conjuntiva. A doente referia como antecedentes relevantes diabetes gestacional, depressão reactiva e hipertensão arterial.

Estava medicada com bisoprolol $10 \mathrm{mg} /$ dia e com mesalazina $1,5 \mathrm{~g}$ bid desde há 1,5 anos, com mexazolam $0,5 \mathrm{mg}$ bid desde há 2 anos e anticonceptivo oral (drospirenona/etinilestradiol 3mg/0,030mg) desde há alguns anos.

A biopsia lesional mostrou bolha na junção dermoepidérmica (JDE), contendo neutrófilos e raros eosinófilos e na derme moderado infiltrado inflamatório polimórfico. A imunofluorescência directa revelou imunorreactividade linear de $\lg A$ na JDE. Do estudo analítico efectuado registou-se a presença de anticorpos antidesmossomas. 


\section{Caso Clínico}
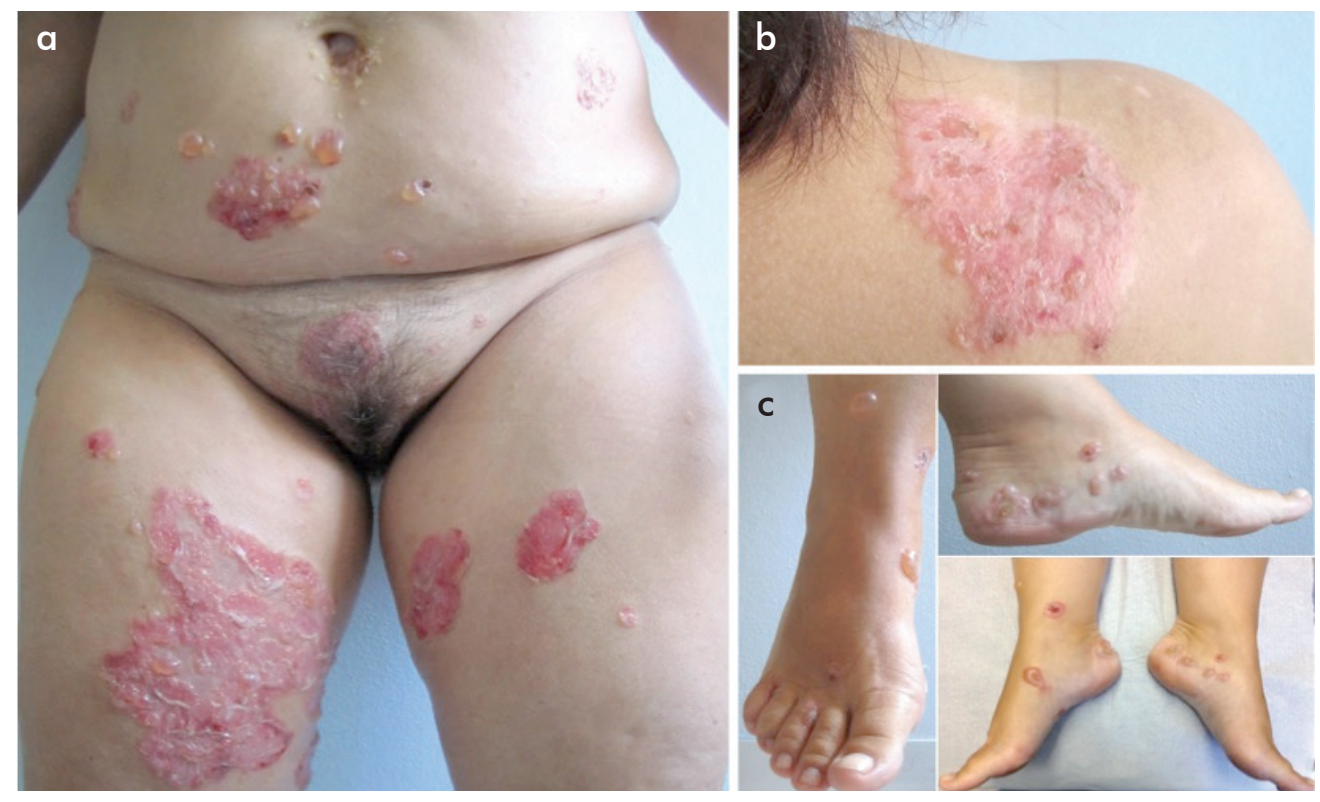

Fig 1 - a) Bolhas tensas, de conteúdo cítrico, em base eritematosa com dimensão compreendida entre 0,5cm e 3cm; b) Placa eritematosa com bolhas e erosões na área supraescapular direita; c) Bolhas tensas na face anterior e medial dos pés.

Foi iniciada prednisolona $1 \mathrm{mg} / \mathrm{kg} /$ dia e dapsona $100 \mathrm{mg} /$ dia. Suspendeu mesalazina. Registou-se uma rápida melhoria clínica e após seis meses de seguimento mantem-se sem lesões, medicada com dapsona $100 \mathrm{mg} /$ dia e prednisolona $10 \mathrm{mg}$ em dias alternados. Não foi reintroduzida a mesalazina, mantendo a $\mathrm{CU}$ controlada.

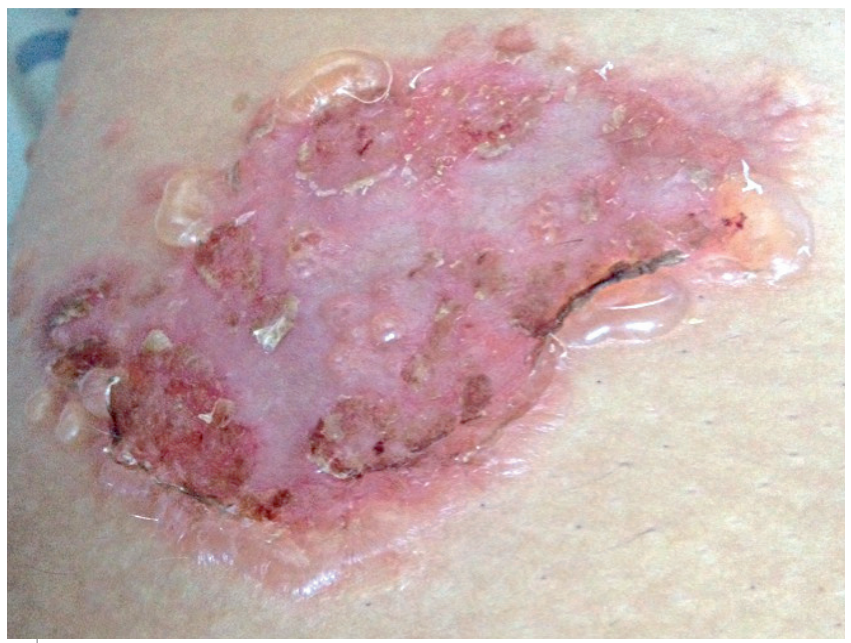

Fig 2 - $5^{\circ}$ dia de internamento - Grande ampliação de lesão de base eritematosa com disposição das bolhas à periferia da mesma - "colar de pérolas".

\section{DISCUSSÃO}

A associação da DBAL com DII ocorre principalmente com a CU, embora a origem desta associação não esteja ainda bem definida. A DBAL é uma doença rara com uma incidência inferior a 0,5 casos/milhão $\left(0,23^{3}\right.$ $0,48^{4}$ casos/milhão). Da análise de 70 doentes com DBAL, encontrou-se a associação com CU em 7,1\% dos doentes, ${ }^{5}$ superior à incidência da $\mathrm{CU}$ na população geral, em Portugal - cerca de 5,5 casos $/ 100000^{6}$. Estão descritos na literatura cerca de 30 casos desta associação. A associação mais frequente da CU com a DBAL, do que com outras dermatoses autoimunes bolhosas, poderá dever-se ao facto de as respostas das mucosas aos antigénios serem frequentemente mediadas por $\lg \mathrm{A}^{2}$. Os plasmócitos na $\mathrm{CU}$ apesar de segregarem uma menor quantidade de $\lg A$, apresentam uma secreção aumentada de $\lg A$ monomérica, $\lg \mathrm{A}_{1}$. Visto a DBAL ser uma doença mediada por $\lg \mathrm{A}_{1}$, esta alteração no perfil imunológico poderá ser relevante para o desenvolvimento da DBAL em indivíduos susceptíveis ${ }^{5}$.

Por outro lado, a inflamação associada à Dll poderá levar a uma reacção ao antigénio BP180 e à indução de uma doença bolhosa mediada pelo BP1803.

A associação da DBAL aos HLA -B8, -DR3, -DQw2 ${ }^{7-9}$ são diferentes da tipagem observada na CU, ao HLA-DR2 ${ }^{10}$ não permitindo estabelecer uma relação genética entre as duas patologias. 


\section{Caso Clínico}

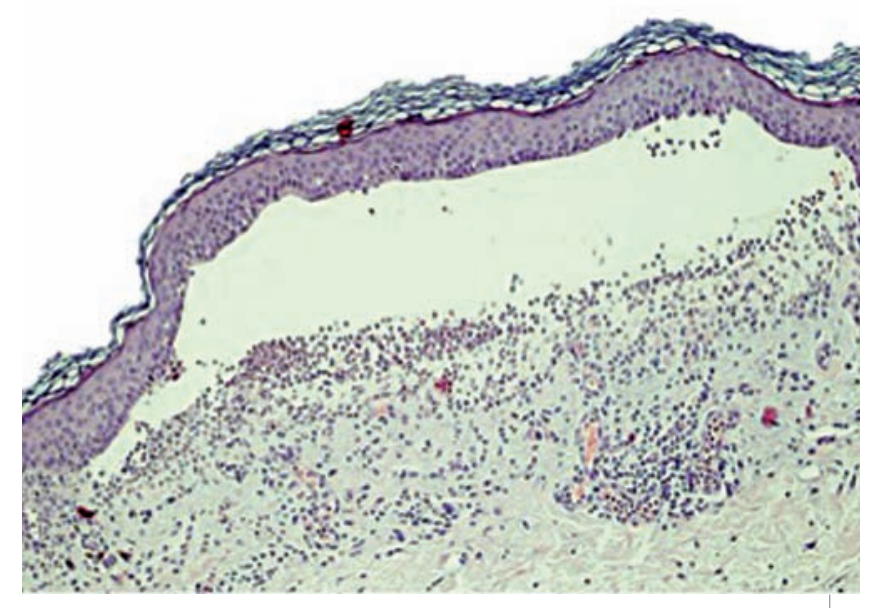

Fig. 3 - Bolha na junção dermoepidérmica contendo neutrófilos e raros eosinófilos e na derme moderado infiltrado inflamatório polimórfico (H\&E, 100x).

A doença intestinal da CU precede habitualmente o aparecimento da $\mathrm{DBAL}^{2}$. A crise de CU poderá funcionar como desencadeante da DBAL, podendo a sua manifestação estar relacionada com a actividade da doença intestinal," tal como aconteceu com a nossa doente.

A eficácia da proctocolectomia na doença cutânea permanece controversa, existindo relatos de melhoria, ${ }^{12}$ mas também de não eficácia da mesma ${ }^{5}$. Há um caso de eficácia na utilização do Infliximab nesta associação ${ }^{13}$.

A existência de uma forma de DBAL induzida por fármacos tem sido reportada, sendo a apresentação clínica por vezes atípica, mais grave, com grandes erosões, com sinal de Nikolsy e envolvimento das mucosas, podendo manifestar-se como DRESS, necrose epidérmica tóxica ou síndrome de Lyell14. A imputabilidade é por vezes difícil sendo mais frequentemente utilizado o score de Naranjo, ${ }^{15}$ podendo ser também útil o método de imputabilidade Francês - algoritmo de Bégaud $^{16}$, muito utilizado na farmacovigilância para reacções cutâneas adversas, não existindo no entanto um método de imputabilidade validado para esta entidade $^{17}$. As características clínicas e histológicas são muito heterogéneas e podem ser completamente indistinguíveis da forma idiopática ${ }^{14,18}$. $O$ período de latência entre o inicio do fármaco e a DBAL pode variar entre os $1-780$ dias, $^{18}$ com uma média de $10^{14}-64^{18}$ dias, sendo esta menor para os casos desencadeados pela vancomicina, despoletados em média 9 dias após o início da toma medicamentosa ${ }^{19}$. O fármaco mais frequentemente associado à DBAL é a vancomicina $^{14,18}$, estando também descritos outros antibióticos, com relevo para as sulfonamidas, como o trimetropim-sulfametoxazol ${ }^{18}$. É de referir, o relato recente de DBAL associado à sulfassalazina manifestando-se como DRESS ${ }^{19}$.

Nesta forma da doença, a resposta à interrupção do fármaco em causa é variável, sendo a remissão clinica completa em $67 \%$ dos casos associados à vancomicina $^{18}$.

A DBAL foi descrita em doentes com CU tratados com sulfassalazina, mesalazina, corticosteróides assim como em doentes que não estavam sob qualquer tratamento farmacológico, sendo improvável que esta associação esteja relacionada com fármacos ${ }^{12}$.

Embora no nosso caso, se tenha realizado terapêutica sem aguardar por eventual resposta após retirada da mesalazina, não se tenha reintroduzido o fármaco suspeito, haja história de um longo período de terapêutica prévio à manifestação da DBAL e as manifestações

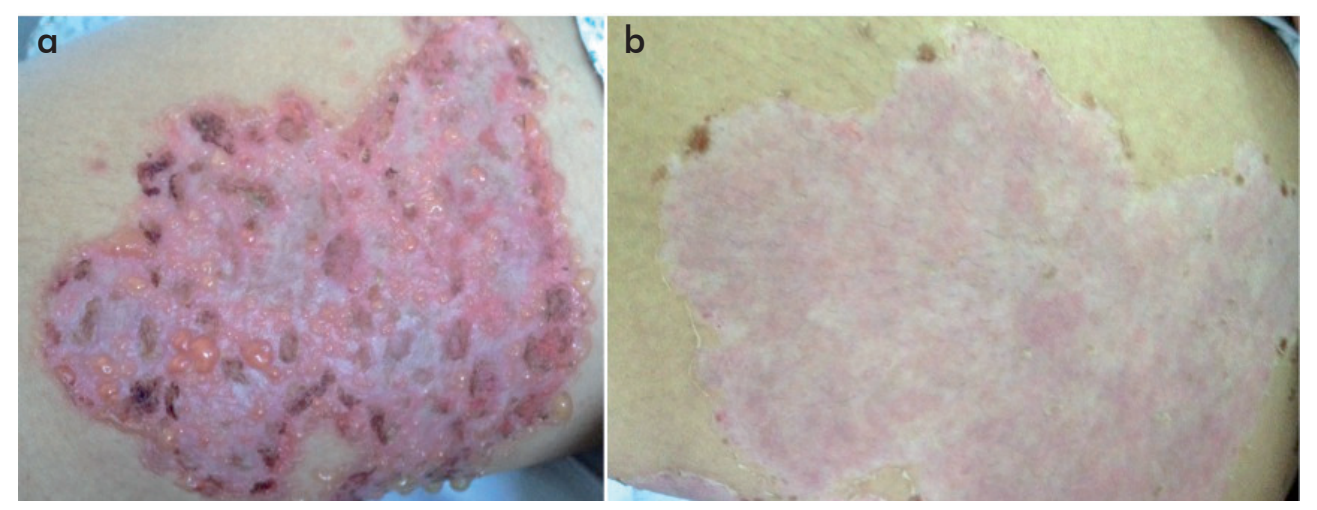

Fig 4 - a) Placa eritematosa com bolhas tensas e erosões de distribuição central e delimitando o bordo da placa, ao $5^{\circ}$ dia de internamento; b) Melhoria da lesão, após 10 dias de tratamento. 
da DBAL não tenham tido maior gravidade que as descritas habitualmente nas formas espontâneas, não pode ser totalmente excluída a imputabilidade da mesalazina na indução da BDAL.

Pretende-se com este caso adicionar à literatura um exemplo de uma associação rara cuja etiologia permanece desconhecida, mas que poderá estar relacionada com a secreção alterada de lgA e sua acção contra os antigénios epidérmicos ou dermoepidérmicos. Relembra-se ainda a existência de uma forma de DBAL associada a fármacos, em constante caracterização.

O aprofundar sobre o conhecimento da patogénese desta associação, possibilitará provavelmente no futuro, uma melhor abordagem a este tipo de patologia, com impacto na avaliação de risco de desenvolvimento da mesma, no prognóstico e no aparecimento de terapêutica dirigida específica.

\section{BIBLIOGRAFIA}

1. Taghipour K, Chi CC, Vincent A, Groves RW, Venning $V$, Wojnarowska $F$. The association of bullous pemphigoid with cerebrovascular disease and dementia: a case-control study. Arch Dermatol 2010; 146: 1251-4.

2. Shipman AR, Reddy H, Wojnarowska F. Association between the subepidermal autoimmune blistering diseases linear $\lg \mathrm{A}$ disease and the pemphigoid group and inflammatory bowel disease: two case reports and literature review. Clin Exp Dermatol. $2012 ; 37(5): 461-8$.

3. Zillikens D, Wever S, Roth A, Weidenthaler-Barth B, Hashimoto T, Bröcker EB. Incidence of autoimmune subepidermal blistering dermatoses in a region of central Germany. Arch Dermatol. 1995; 131: 9578.

4. Bernard P, Vaillant L, Labeille B, Bedane C, Arbeille $B$, Denoeux JP, et al. Incidence and distribution of subepidermal utoimmune bullous skin diseases in three French regions. Bullous Diseases French Study Group. Arch Dermatol. 1995; 131: 48-52.

5. Paige DG, Leonard JN, Wojnarowska F, Fry L. Linear IgA disease and ulcerative colitis. Br J Dermatol. $1997 ; 136: 779-82$.

6. Shivananda S, Lennard-Jones J, Logan R, Fear N, Price A, Carpenter $L$, et al. Incidence of inflammatory bowel disease across Europe: is there a difference between north and south? Results of the European collaborative study on inflammatory bowel disease (EC-IBD). Gut. 1996; 39:690-7.
7. Leonard JN, Haffenden GP. Ring NP, McMinn RM, Sidgwick A, Mowbray JF, et al Linear IgA disease in adults. Br J Dermatol. 1982; 107: 301-16.

8. Collier PM, Wojnarowska F, Bhogal B. Class I and class II HLA antigens in chronic bullous dermatosis of childhood. Br J Dermatol. 1991; 125: 486.

9. Collier PM, Wojnarowska F. MHC class I and II antigens in linear IgA dermatosis. J Invest Dermatol. 1992; 98: 526.

10. Toyoda H, Wang SJ, Yang H-Y, Redford A, Magalong $D$, Tyan $D$, et al Distinct associations of HLA class II genes with inflammatory bowel disease. Gastroenterology. 1993; 104:741-8.

11. Taniguchi T, Maejima H, Saito N, Katsuoka K, Haruki S. Case of linear IgA bullous dermatosis-involved ulcerative colitis. Inflamm Bowel Dis. 2009; 15(9):1284-5.

12. Caldarola G, Annese V, Bossa F, Pellicano R. Linear IgA bullous dermatosis and ulcerative colitis treated by proctocolectomy. Eur J Dermatol. 2009; 19:651.

13. Yamada S, Makino T, Jinnin M, Sakai K, Fukushima $S$, Inove $Y$, et al. Association of linear $\lg A$ bullous disease with ulcerative colitis: a case of successful treatment with infliximab. Dermatology. 2013; 227(4):295-8.

14. Chanal J, Ingen-Housz-Oro S, Ortonne N, Duong TA, Thomas $M$, Valeyrie-Allanore $L$, et al. Linear $\lg A$ bullous dermatosis: comparison between the drug-induced and spontaneous forms. Br J Dermatol. 2013; 169(5):1041-8.

15. Naranjo CA, Busto U, Sellers EM, Sandor P, Ruiz I, Roberts EA, et al. A method for estimating the probability of adverse drug reactions. Clin Pharmacol Ther. 1981; 30:239-45.

16. Bégaud B, Evreux JC, Jouglard J, Lagier G. Imputation of the unex- pected or toxic effects of drugs. Actualization of the method used in France. Therapie. 1985; 40:111-8.

17. Benahmed S, Picot M-C, Dumas F, Demoly P. Accuracy of a pharmacovigilance algorithm in diagnosing drug hypersensitivity reactions. Arch Intern Med. 2005; 165:1500-5.

18. Fortuna G, Salas-Alanis JC, Guidetti E, Marinkovich MP. A critical reappraisal of the current data on drug-induced linear immunoglobulin A bullous dermatosis: a real and separate nosological entity? J Am Acad Dermatol. 2012; 66(6):988-94

19. Hernández N, Borrego L, Soler E, Hernández J. Sulfasalazine-induced linear immunoglobulin A bullous dermatosis with DRESS. Actas Dermosifiliogr. $2013 ; 104(4)$ : 343-6. 